“(C) 2021 IEEE. Personal use of this material is permitted. Permission from IEEE must be obtained for all other uses, in any current or future media, including reprinting/republishing this material for advertising or promotional purposes, creating new collective works, for resale or redistribution to servers or lists, or reuse of any copyrighted component of this work in other works." 


\title{
A Sidelobe Suppressed Huygens Dipole Array with High Aperture Efficiency
}

\author{
Wei Lin and Richard W. Ziolkowski \\ Global Big Data Technologies Centre, \\ School of Electrical and Data Engineering, \\ University of Technology Sydney, \\ Ultimo 2007, Sydney, Australia, \\ Email:Wei.Lin@uts.edu.au, Richard.Ziolkowski@uts.edu.au
}

\begin{abstract}
A sidelobe suppressed, high aperture efficiency Xband Huygens dipole array is reported. The Huygens array consists of four magnetic and four electric dipole radiators. A collinear set of magnetic dipoles (MDs) is formed from a $\mathbf{T E}_{\mathbf{0 . 5 , 0}}$ mode open waveguide integrated with phase inverters. The electric dipoles (EDs) are produced by connecting two metallic plates to the aperture of the waveguide, one on each side of it and orthogonal to that vertical wall of the waveguide. The consequent pairs of MDs and EDs are orthogonally oriented and their phase difference is a natural $90^{\circ}$. These dipoles are excited by an electric pin probe inserted into the waveguide at its center through one vertical wall. The overlapped -10-dB impedance and 3-dB realized gain bandwidth is $800 \mathrm{MHz}$, covering 9.45 to $10.25 \mathrm{GHz}$. The sidelobe levels of the $1 \times 4$ array are significantly reduced to $-23.3 \mathrm{~dB}$ thanks to the natural tapering of the amplitude distributions of the MDs and EDs from the center of the array to both of its ends. The peak realized gain value is $12 \mathrm{dBi}$. The aperture efficiency is $\mathbf{7 4 \%}$.
\end{abstract}

Index Terms-Antenna arrays, aperture efficiency, backlobes, electric dipole, Huygens sources, magnetic dipole, sidelobes.

\section{INTRODUCTION}

Sidelobe level suppression of an antenna array is critical as high sidelobes will create unwanted signal interferences and energy waste. In particular, wireless applications like radar systems [1] and radio telescopes [2] require sidelobe levels to be less than $-20 \mathrm{~dB}$. It is well known that the first sidelobe level of a uniform linear antenna array is $-13.26 \mathrm{~dB}$ if each of its elements is excited with equal amplitude and phase. One effective and popular method to suppress the sidelobe levels of the fields radiated by an antenna array is to excite its elements with a tapered amplitude distribution, i.e., with amplitudes decreasing from its central ones to those on its edges. However, as a consequence, the resultant aperture efficiency decreases. It is challenging to design and realized an antenna array with both low sidelobe levels and a high aperture efficiency.

Many efforts have been made to suppress the sidelobe levels of antenna arrays as reported in [3] - [10]. The associated design methodologies can be categorized into two general classes. The first is to design an array of identical radiating elements that are excited with a feed network which delivers tapered amplitudes to them [3] - [6]. The second one is design an array of appropriately adjusted radiating elements that are fed by a network which outputs an equal amplitude and phase to each of them [7] - [10]. All of the noted arrays in either class can achieve sidelobe levels that are smaller than $20 \mathrm{~dB}$ from the peak value of the main lobe. However, they all exhibit a maximum aperture efficiency that is less than $50 \%$.

This paper reports, as will the ensuing presentation, an innovative X-band Huygens antenna array that simultaneously achieves a broadside beam with high directivity, low sidelobe levels, and a high aperture efficiency. Four collinear in-phase magnetic dipoles (MDs) and four orthogonal collinear in-phase electric dipoles (EDs) are realized in a compact entity. The MDs are produced by a $\mathrm{TE}_{0.5,0}$ mode open waveguide with integrated phase inverters. The EDs are realized by connecting two metallic plates to the waveguide aperture, one on each side of it and oriented orthogonal to that vertical wall. These MDs and EDs are orthogonal and their phase difference is a natural $90^{\circ}$. Each pair consequently forms a Huygens dipole element. The resulting $1 \times 4$ antenna array of Huygens dipole elements is excited at the center of its waveguide by an electric pin probe that is inserted through one of its vertical walls and is driven via a microstrip transition. The overlapped $-10-\mathrm{dB}$ impedance and 3-dB realized gain bandwidth covers $800 \mathrm{MHz}$, from 9.45 to $10.25 \mathrm{GHz}$. Its sidelobe level is $-23.3 \mathrm{~dB}$, and its aperture efficiency reaches $74 \%$.

\section{ANTENNA DESIGN}

The Huygens dipole antenna array configuration is illustrated in Fig. 1. It consists of three main parts. A $1 \times 4$ omnidirectional magnetic dipole (OMD) array is facilitated by an open $\mathrm{TE}_{0.5,0}$ mode waveguide with two phase inverters. The OMD array consists of four half-wavelength long sections and is fabricated from a single piece of copper-clad $1.58 \mathrm{~mm}$ thick Rogers Duroid ${ }^{\mathrm{TM}} 5880$ substrate, whose relative permittivity and loss tangent are 2.2 and 0.0009 , respectively. Details of the OMD array design concept are found in our previously reported work [11]. Two metallic dipole plates are connected directly to the edges of the waveguide aperture and are orthogonal to its vertical walls. The four ensuing EDs occur over the same four waveguide sections associated with the MDs. Finally, a microstrip feedline with an impedance transformer is attached on one of the vertical walls of the 
waveguide. It matches the impedance of the Huygens array to that of a $50-\Omega$ SMA connector. This feedline is realized with the same Rogers Duroid ${ }^{\mathrm{TM}} 5880$ substrate, but with a 0.508 $\mathrm{mm}$ thickness. The entire volume of the array is compact, $L \times$ $W \times H=68.0 \times 22.58 \times 6.0 \mathrm{~mm}^{3}\left(2.27 \times 0.75 \times 0.2 \lambda_{0}{ }^{3}\right.$ at the $\mathrm{X}$-band operating frequency, $10 \mathrm{GHz}$ ).
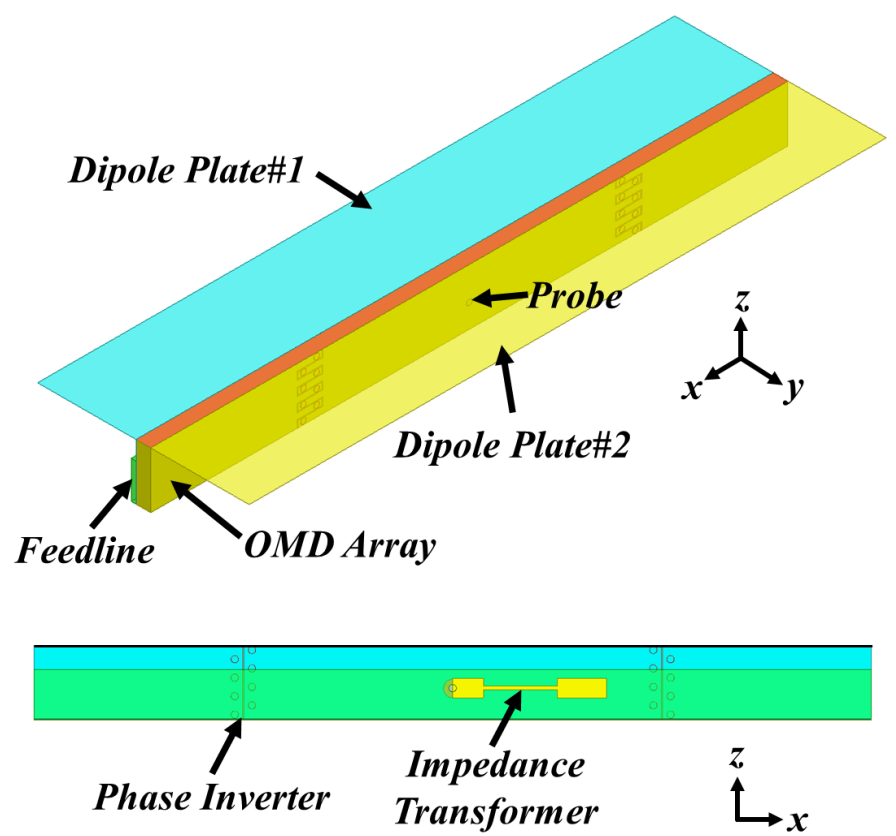

Fig. 1. Configuration of the Huygens dipole array with low sidelobe levels. (Upper) Perspective view. (Below) Side view.

\section{OPERATING PRINCIPLE AND PERFORMANCE}

Huygens radiation is produced by each MD-ED pair and the entire developed antenna array. It has been extensively studied that a pair of orthogonal and in-phase MD and ED current densities will produce a Huygens cardioid pattern [12] - [15]. The operating mechanism of the array is clearly demonstrated with the electric and magnetic fields distributions presented in Fig. 2. Top views of the E-field distributions on the two metallic plates at two quarter periods of time at $10 \mathrm{GHz}$ are shown in the upper two sub-plots. Side views of the corresponding $\mathrm{H}$-field distributions are found in the bottom two sub-plots. It is clearly observed that the EDs formed on the two plates and the MDs formed in the waveguide aperture resonate alternately in each quarter period of time. As the magnetic currents have a $90^{\circ}$ phase difference ahead of the electric currents, the MDs and EDs radiate in-phase. Notice in particular that the amplitudes of both the MDs and EDs are naturally tapered from the center of the array to its ends. Without any ground plane, the unidirectional cardioid realized gain pattern shown in Fig. 3(a) demonstrates that the fourelement Huygens dipole array achieves a broadside main beam having a very broad beamwidth and very low sidelobe levels. In comparison, Fig. 3(b) demonstrates that an omnidirectional realized gain pattern is radiated by the corresponding $1 \times 4$ array of MDs that results when the two plates are removed.
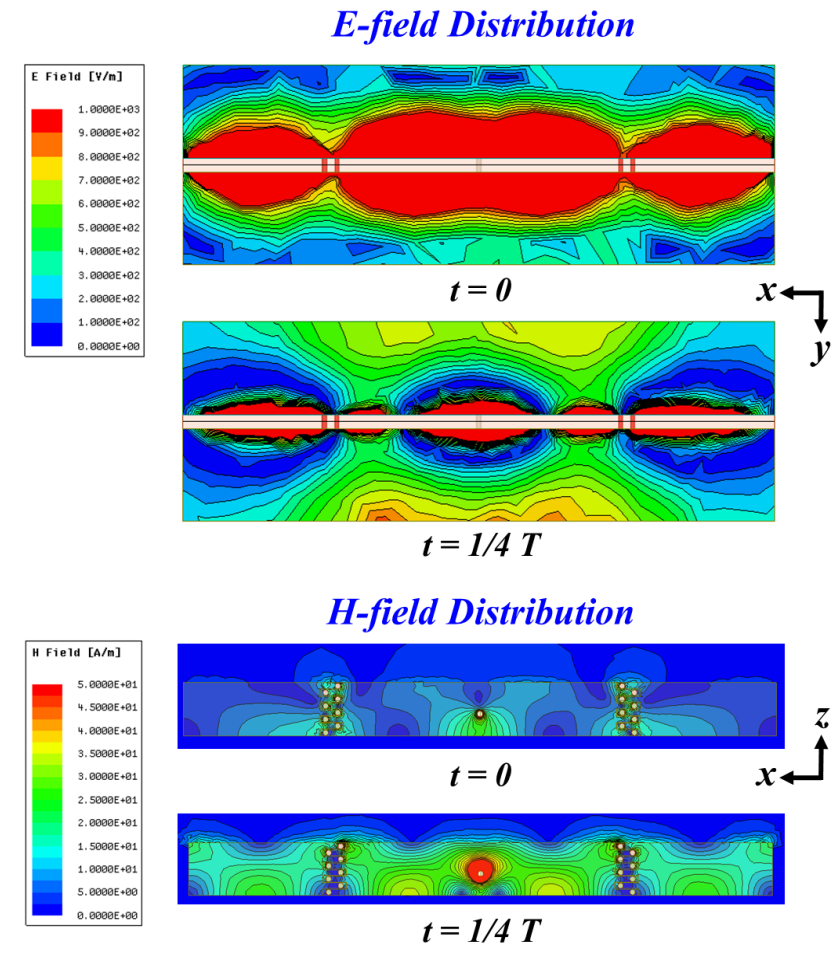

Fig. 2. Electric field distribution on the two dipole plates and the magnetic field distribution in the $\mathrm{TE}_{0.5,0}$ mode waveguide at two quarter periods of time at $10.0 \mathrm{GHz}$.

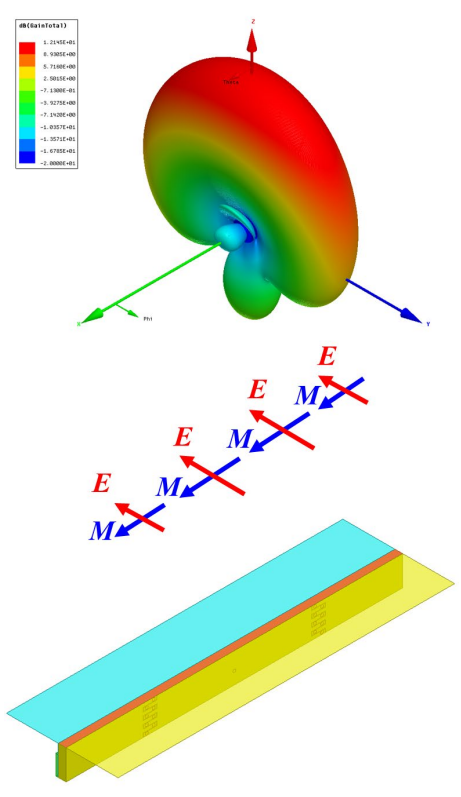

(a)
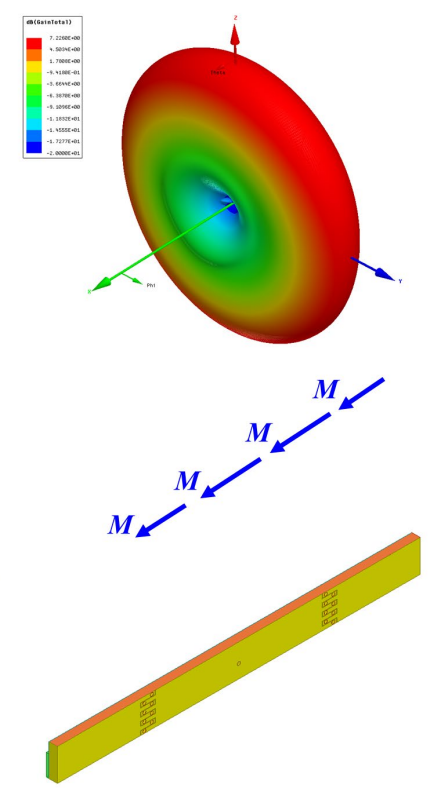

(b)

Fig. 3. Comparison of the simulated realized gain patterns of two arrays, their respective ED and MD sources, and their 3D models. (a) Huygens dipole array (plates present). (b) Magnetic dipole array (plates absent). 
The $\left|\mathrm{S}_{11}\right|$ and realized gain values of the $1 \times 4$ Huygens dipole array are shown in Fig. 4(a) as functions of the source frequency. The overlapped $-10-\mathrm{dB}$ impedance and 3-dB realized gain bandwidth covers $800 \mathrm{MHz}$ band from 9.45 to 10.25 GHz. The peak realized gain is $12 \mathrm{dBi}$. The aperture efficiency reaches $74 \%$; it is the highest amongst the reported arrays with low sidelobe levels in [3] - [10]. The realized gain patterns at $10 \mathrm{GHz}$ in the two principal vertical planes are shown in Fig. 4(b). It is clearly seen that the sidelobe levels are suppressed below $-23 \mathrm{~dB}$ from the peak broadside value. A wide beamwidth, $87^{\circ}$, is found in the H-plane $\left(\varphi=90^{\circ}\right)$ orthogonal to the length of the array and a narrow beamwidth $\left(24^{\circ}\right)$ is identified in the E-plane $\left(\varphi=0^{\circ}\right)$ along it.

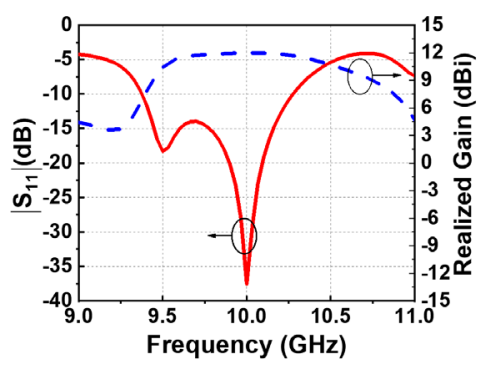

(a)

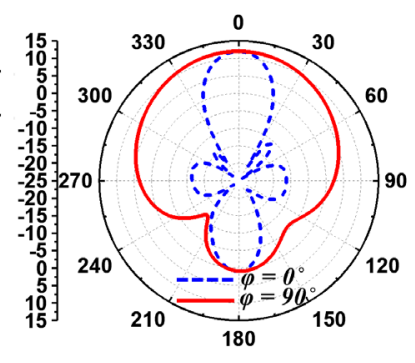

(b)
Fig. 4. Huygens dipole antenna array performance. (a) $\left|S_{11}\right|$ and realized gain values as functions of the source frequency. (b) Realized gain patterns at 10 $\mathrm{GHz}$ in the two principal vertical planes.

\section{CONCLUSIONS}

An innovative X-band $1 \times 4$ Huygens dipole antenna array was presented. Its performance characteristics were summarized and are highly attractive. The array has a number of potential uses for $5 \mathrm{G}$ wireless applications including being the source for Internet-of-things (IoT) devices energized by wireless power transfer (WPT).

\section{REFERENCES}

[1] J. N. Sahalos, "Design of shared aperture radar arrays with low sidelobe level of the two-way array factor," IEEE Trans. Antennas Propag., vol. 68 , no. 7, pp. 5415-5420, July 2020.

[2] J. Teniente, R. Gonzalo, and C. D. Rio, "Low sidelobe corrugated horn antennas for radio telescopes to maximize $\mathrm{G} / \mathrm{T}_{\mathrm{s}}$," IEEE Trans. Antennas Propag., vol. 59, no. 6, pp. 1886-1893, June 2011.

[3] G. L. Huang, S. G. Zhou, T. H. Chio, H. T. Hui, and T. S. Yeo "A low profile and low sidelobe wideband slot antenna array fed by an amplitude-tapering waveguide feed-network," IEEE Trans. Antennas Propag., vol. 63, no. 1, pp. 419-423, Jan. 2015.

[4] L. Chang, Y. Li, Z. Zhang, X. Li, S. Wang and Z. Feng, "Low-sidelobe air-filled slot array fabricated using silicon micromachining technology for millimeter-wave application," IEEE Trans. Antennas Propag., vol. 65 , no. 8, pp. 4067-4074, Aug. 2017.

[5] Y. J. Cheng, J. Wang and X. L. Liu, “:94 GHz substrate integrated waveguide dual-circular-polarization shared-aperture parallel-plate longslot array antenna with low sidelobe level," IEEE Trans. Antennas Propag., vol. 65, no. 11, pp. 5855-5861, Nov. 2017.

[6] H. Chu, P. Li and Y. X. Guo "A Beam-Shaping Feeding Network in Series Configuration for Antenna Array With Cosecant-Square Pattern and Low Sidelobes," IEEE Antennas Wireless Propag. Lett., vol. 18, no. 4, pp. 742-746, Apr. 2019.

[7] J. Yin, Q. Wu, C. Yu, H. Wang and W. Hong, "Low-sidelobe-level series-fed microstrip antenna array of unequal interelement spacing," IEEE Antennas Wireless Propag. Lett., vol. 16, pp. 1695-1698, 2017.

[8] Y. Geng, J. Wang, Y. Li, Z. Li, M. Chen and Z. Zhang, "High-efficiency leaky-wave antenna array with sidelobe suppression and multibeam generation," IEEE Antennas Wireless Propag. Lett., vol. 16, pp. 2787$2790,2017$.

[9] Y. W. Wu, Z. Jiang and Z. C, Hao, "A 400-GHz low cost planar leakywave antenna with low sidelobe level and low cross-polarization level," IEEE Trans. THz Sci. Technol., vol. 10, no. 4, pp. 427-430, Jul. 2020.

[10] S. Ogurtsov and S. Koziel, "On alternative approaches to design of corporate feeds for low-sidelobe microstrip linear arrays," IEEE Trans. Antennas Propag., vol. 66, no. 7, pp. 3781-3786, Jul. 2018.

[11] W. Lin and R. W. Ziolkowski, "High-directivity, compact, omnidirectional horizontally polarized antenna array," IEEE Trans. Antennas Propag., vol. 68, no. 8, pp. 6049-6058, Apr. 2020.

[12] K.-M. Luk and H. Wong, "A new wideband unidirectional antenna element," Int. J. Microw. Opt. Technol., vol. 1, no. 1, pp. 35-44, Jun. 2006.

[13] W. Lin and R. W. Ziolkowski, "Electrically-small, low-profile, Huygens circularly polarized antenna," IEEE Trans. Antennas Propag., vol. 66, no. 2, pp. 636-643, Feb. 2018.

[14] R. W. Ziolkowski, "Using Huygens multipole arrays to realize unidirectional needle-like radiation," Phys. Rev. X, vol. 7, 031017, Jul. 2017.

[15] W. Lin, and R. W. Ziolkowski, "Electrically small, single-substrate Huygens dipole rectenna for ultra-compact wireless power transfer applications," IEEE Trans. Antennas Propag., in Early Access, Oct. 2020. DOI: 10.1109/TAP.2020.3004987. 